\title{
Lie Group Analysis of Mixed Convection Flow with Mass Transfer over a Stretching Surface with Suction or Injection
}

\author{
Mudassar Jalil, Saleem Asghar, and Muhammad Mushtaq \\ Department of Mathematics, COMSATS Institute of Information Technology, H-8, \\ 44000 Islamabad, Pakistan \\ Correspondence should be addressed to Mudassar Jalil, mudassarjalil@yahoo.com
}

Received 25 May 2010; Revised 27 August 2010; Accepted 14 October 2010

Academic Editor: Cristian Toma

Copyright (C) 2010 Mudassar Jalil et al. This is an open access article distributed under the Creative Commons Attribution License, which permits unrestricted use, distribution, and reproduction in any medium, provided the original work is properly cited.

\begin{abstract}
The mixed convection flow with mass transfer over a stretching surface with suction or injection is examined. By using Lie group analysis, the symmetries of the equations are calculated. A four-finite parameter and one infinite parameter Lie group transformations are obtained. Two different cases are discussed, one for the scaling symmetry and the other for spiral symmetry. The governing partial differential equations are transformed into ordinary differential equations using these symmetries. It has been noted that the similarity variables and functions available in the literature become special cases of the similarity variables and functions discussed in this paper.
\end{abstract}

\section{Introduction}

The study of continuously stretching sheets has many applications in manufacturing industries. Application of stretching sheets can be found in the areas like paper production, hot rolling, glass blowing, continuous casting of metals, and wire drawing. First of all Sakiadis $[1,2]$ investigated the boundary layer behavior on stretching surfaces and presented numerical solution for the sheet having constant speed. Extension to this problem where velocity is proportional to the distance from the slit was given by Crane [3]. Flow and heat transfer in the boundary layer on stretching surface was studied by Tsou et al. [4]. Fox et al. [5] presented different methods (analytical or numerical) for solving problems of stretching sheet with suction and injection. Heat and mass transfer on stretched surface with suction and injection was introduced by Erickson et al. [6]. P. S. Gupta and A. S. Gupta [7] studied the same problem for linearly stretching sheet. Heat transfer past a moving continuous plate with variable temperature was studied by Soundalgekar and Murty [8] and Grubka et al. [9]. Ali [10] presented similarity solutions for stretched surface with suction and injection. Hayat et al. [11] investigated the effect of heat and mass transfer for Soret and Dufour's effect on 
mixed convection boundary layer flow over a stretching vertical surface in a porous medium filled with a viscoelastic fluid.

Lie group analysis is a classical method discovered by Norwegian mathematician Sophus Lie for finding invariant and similarity solutions [12-15]. Yürüsoy and Pakdemirli [16] presented exact solution of boundary layer equations of a special non-Newtonian fluid over a stretching sheet by the method of Lie group analysis. They extended their work to creeping flow of second-grade fluid [17]. Sivasankaran et al. [18, 19] studied the problem of natural convection heat and mass transfer flow past an inclined plate for various parameters using Lie group analysis without and with heat generation.

In this paper, Lie group analysis of mixed convection flow with mass transfer over a stretching surface with suction or injection is studied. Here the application of Lie group analysis for the fluid flow problem is discussed, with the help of examples from the literature, in such a way that one can easily understand its importance for finding similarity variables and functions. There are two important features of this work that make it different from the other. First, instead of taking a specific form of the boundary conditions (i.e., stretching velocity of the surface, wall temperature, and concentration), these are taken as arbitrary functions. Second, symmetry operator is applied not only to the equations of the problem but also to the boundary conditions of the problem in such a way that with infinite parameter Lie group transformations those finite parameters from infinitesimals are also discarded under which boundary conditions fail to be invariant. It has been wiliness that the number of symmetries reduce in order to comply with the invariance of boundary conditions. It is shown that mixed convection flow with mass transfer is possible only for two types of vertical stretching, the polynomial stretching and the exponential stretching. From examples it is shown that the similarities of the problems discussed by Ali and Al-Yousef [20], Yih [21], and Chen [22] become special subcases of the case of polynomial stretching and the problem discussed by Partha et al. [23] becomes a special subcase of the exponential stretching case. In the case of polynomial stretching a new example is included.

\section{Equations of Motion}

Consider a two-dimensional laminar flow of a steady incompressible viscous fluid over a permeable surface stretching with velocity $u_{w}(\tilde{x})$. The temperature distribution and concentration at the surface are $T_{w}(\tilde{x})$ and $C_{w}(\tilde{x})$, respectively. The surface is moving through a quiescent ambient fluid with constant temperature $\widetilde{T}_{\infty}$ and constant concentration $\widetilde{C}_{\infty}$. $v_{w}(\tilde{x})$ is the suction or injection velocity through the surface. Here we do not assume a specific form of velocity, temperature, and concentration variations for the stretching surface. Using Boussinesq approximations, the governing equations for the boundary layer flows over a stretching surface with suction or injection are expressed as

$$
\begin{gathered}
\frac{\partial \tilde{u}}{\partial \tilde{x}}+\frac{\partial \widetilde{v}}{\partial \tilde{y}}=0 \\
\tilde{u} \frac{\partial \tilde{u}}{\partial \tilde{x}}+\widetilde{v} \frac{\partial \tilde{u}}{\partial \tilde{y}}=v \frac{\partial^{2} \tilde{u}}{\partial \tilde{y}^{2}}+g \beta\left(\widetilde{T}-\widetilde{T}_{\infty}\right)+g \beta^{*}\left(\tilde{C}-\tilde{C}_{\infty}\right) \\
\tilde{u} \frac{\partial \tilde{T}}{\partial \tilde{x}}+\widetilde{v} \frac{\partial \tilde{T}}{\partial \tilde{y}}=\alpha \frac{\partial^{2} \tilde{T}}{\partial \tilde{y}^{2}} \\
\tilde{u} \frac{\partial \tilde{C}}{\partial \tilde{x}}+\tilde{v} \frac{\partial \tilde{C}}{\partial \tilde{y}}=D \frac{\partial^{2} \tilde{C}}{\partial \tilde{y}^{2}}
\end{gathered}
$$


along with the following boundary conditions:

$$
\begin{gathered}
\tilde{u}=U_{0} u_{w}(\tilde{x}), \quad \tilde{T}-\tilde{T}_{\infty}=T_{0} T_{w}(\tilde{x}), \\
\tilde{v}=V_{0} v_{w}(\tilde{x}), \quad \tilde{C}-\tilde{C}_{\infty}=C_{0} C_{w}(\tilde{x}) \quad \text { at } \tilde{y}=0, \\
\tilde{u} \longrightarrow 0, \quad \tilde{T} \longrightarrow \tilde{T}_{\infty}, \quad \tilde{C} \longrightarrow \tilde{C}_{\infty} \text { as } \tilde{y} \longrightarrow \infty,
\end{gathered}
$$

where $\tilde{u}$ and $\tilde{v}$ are the components of velocity in $\tilde{x}$ and $\tilde{y}$ directions, $v$ is the fluid kinematic viscosity, $g$ is the gravitational acceleration, $\beta$ is the coefficient of thermal expansion, $\beta^{*}$ is the coefficient of concentration expansion, $\alpha$ is the thermal diffusivity, and $D$ is the mass diffusivity. $U_{0}$ and $V_{0}$ are the reference velocities, whereas $T_{0}$ and $C_{0}$ are the reference temperature and mass concentration, respectively.

Introducing the nondimensional parameters

$$
\begin{array}{cc}
x=\frac{\tilde{x}}{L}, \quad y=\frac{\tilde{y}}{L}\left(\frac{U_{0} L}{v}\right)^{1 / 2}, \quad u=\frac{\tilde{u}}{U_{0}}, \\
v=\frac{\tilde{v}}{U_{0}}\left(\frac{U_{0} L}{v}\right)^{1 / 2}, \quad T=\frac{\left(\widetilde{T}-\tilde{T}_{\infty}\right)}{T_{0}}, \quad C=\frac{\left(\widetilde{C}-\tilde{C}_{\infty}\right)}{C_{0}},
\end{array}
$$

in (2.1), one gets

$$
\begin{gathered}
\frac{\partial u}{\partial x}+\frac{\partial v}{\partial y}=0 \\
u \frac{\partial u}{\partial x}+\mathrm{v} \frac{\partial u}{\partial y}=\frac{\partial^{2} u}{\partial y^{2}}+\frac{g \beta T_{0} L}{U_{0}^{2}} T+\frac{g \beta^{*} C_{0} L}{U_{0}^{2}} C \\
u \frac{\partial T}{\partial x}+v \frac{\partial T}{\partial y}=\frac{1}{\operatorname{Pr}} \frac{\partial^{2} T}{\partial y^{2}} \\
u \frac{\partial C}{\partial x}+v \frac{\partial C}{\partial y}=\frac{1}{\mathrm{Sc}} \frac{\partial^{2} C}{\partial y^{2}}
\end{gathered}
$$

where $\operatorname{Pr}=v / \alpha$ is the Prandtl number and $\mathrm{Sc}=v / D$ is the Schmidt number. Boundary conditions for the above equations are

$$
\begin{aligned}
& y=0 ; u=u_{w}(x), \quad v=\frac{V_{0} v_{w}(x)}{U_{0}}\left(\frac{U_{0} L}{v}\right)^{1 / 2}, \\
& T=T_{w}(x), \quad C=C_{w}(x), \\
& y \longrightarrow \infty ; \quad u=0, \quad T=0, \quad C=0 .
\end{aligned}
$$




\section{Symmetries of the Problem}

By applying Lie group method [12] to (2.4)-(2.7), the infinitesimal generator for the problem can be written as

$$
X=\xi_{1} \frac{\partial}{\partial x}+\xi_{2} \frac{\partial}{\partial y}+\phi_{1} \frac{\partial}{\partial u}+\phi_{2} \frac{\partial}{\partial v}+\phi_{3} \frac{\partial}{\partial T}+\phi_{4} \frac{\partial}{\partial C}
$$

One requires that the equations remain invariant under the infinitesimal Lie point transformations

$$
\begin{aligned}
& x^{*}=x+\epsilon \xi_{1}(x, y, u, v, T, C)+O\left(\epsilon^{2}\right), \\
& y^{*}=y+\epsilon \xi_{2}(x, y, u, v, T, C)+O\left(\epsilon^{2}\right), \\
& u^{*}=u+\epsilon \phi_{1}(x, y, u, v, T, C)+O\left(\epsilon^{2}\right), \\
& v^{*}=v+\epsilon \phi_{2}(x, y, u, v, T, C)+O\left(\epsilon^{2}\right), \\
& T^{*}=T+\epsilon \phi_{3}(x, y, u, v, T, C)+O\left(\epsilon^{2}\right), \\
& C^{*}=C+\epsilon \phi_{4}(x, y, u, v, T, C)+O\left(\epsilon^{2}\right) .
\end{aligned}
$$

By employing a tedious and straightforward algebra, the form of the infinitesimals is

$$
\begin{gathered}
\xi_{1}=c+d x, \quad \xi_{2}=b y+\gamma(x), \quad \phi_{1}=(-2 b+d) u, \\
\phi_{2}=-b v+u \gamma^{\prime}(x), \quad \phi_{3}=-e+(-4 b+d) T, \\
\phi_{4}=\frac{\beta T_{0}}{\beta^{*} C_{0}} e+(-4 b+d) C .
\end{gathered}
$$

Therefore, the equations admit four finite parameter Lie group transformations. $\gamma(x)$ is infinite parameter Lie group transformation. Parameter $c$ corresponds to the translation in the variable $x$, and parameter $e$ corresponds to the translation in the variables $T$ and $C$. Parameter $b$ corresponds to scaling in the variables $y, u, v, T$, and $C$. Parameter $d$ corresponds to the scaling in the variables $x, u, T$, and $C$. In the following sections, similarity variables and functions corresponding to the above symmetries will be derived.

Before finding similarity variables and functions for all of the symmetries of the problem, the symmetry operator $X$ will be applied to the boundary conditions and then only those symmetries will be discussed that leave the boundary conditions invariant. 


\section{Invariance of the Boundary Conditions}

By applying the symmetry operator $X$, that is defined in (3.1), on boundary conditions, we get differential equations

$$
\begin{gathered}
\frac{\partial u_{w}}{\partial x}-\frac{(-2 b+d)}{(c+d x)} u_{w}=0 \\
\frac{\partial v_{w}}{\partial x}+\frac{b}{(c+d x)} v_{w}=0 \\
\frac{\partial T_{w}}{\partial x}-\frac{(-4 b+d)}{(c+d x)} T_{w}=0 \\
\frac{\partial C_{w}}{\partial x}-\frac{(-4 b+d)}{(c+d x)} C_{w}=0
\end{gathered}
$$

with some restrictions $e=\gamma(x)=0$.

Therefore, the possible boundary conditions for the current problem are as follows: for $d \neq 0$,

$$
\begin{gathered}
u_{w}(x)=A_{1}(c+d x)^{(-2 b+d) / d}, \quad v_{w}(x)=A_{2}(c+d x)^{-b / d}, \\
T_{w}(x)=\mathrm{A}_{3}(c+d x)^{(-4 b+d) / d}, \quad C_{w}(x)=A_{4}(c+d x)^{(-4 b+d) / d} .
\end{gathered}
$$

For $d=0$ and $c \neq 0$,

$$
\begin{array}{cc}
u_{w}(x)=A_{5} \exp \left(-\frac{2 b}{c} x\right), & v_{w}(x)=A_{6} \exp \left(-\frac{b}{c} x\right), \\
T_{w}(x)=A_{7} \exp \left(-\frac{4 b}{c} x\right), & C_{w}(x)=A_{8} \exp \left(-\frac{4 b}{c} x\right),
\end{array}
$$

where $A_{i}$ for $i=1,2,3, \ldots, 8$ are constants of integration.

\section{Determining Similarity Variables and Functions}

From (4.2) and (4.3) it is shown that only two types of vertical stretching are possible: polynomial stretching for $d \neq 0$ and exponential stretching for $d=0$. In the following work each case will be described with examples.

\subsection{Case 1: For $d \neq 0$}

In this case, the parameters $b, c$, and $d$ are taken as arbitrary and $e=\gamma(x)=0$ is considered. This case is combination of scaling and translational symmetry. For $c=0$ it becomes a scaling symmetry. 

be

The characteristic equations for obtaining the similarity transformations would then

$$
\frac{d x}{(c+d x)}=\frac{d y}{b y}=\frac{d u}{(-2 b+d) u}=\frac{d v}{-b v}=\frac{d T}{(-4 b+d) T}=\frac{d C}{(-4 b+d) C} .
$$

The resulting similarity variable is

$$
\eta=\frac{y}{K_{1}^{1 / 2}(c+d x)^{b / d}}
$$

and the similarity functions are

$$
\begin{array}{cc}
u=(c+d x)^{(-2 b+d) / d} F^{\prime}(\eta), & v=(c+d x)^{-b / d} H(\eta), \\
T=(c+d x)^{(-4 b+d) / d} \theta(\eta), & C=(c+d x)^{(-4 b+d) / d} \varphi(\eta) .
\end{array}
$$

From (4.2) and (5.3), we have for $A_{i}=1(i=1,2,3,4)$

$$
\begin{array}{cc}
u=u_{w}(x) F^{\prime}(\eta), & v=-v_{w}(x) H(\eta), \\
T=T_{w}(x) \theta(\eta), & C=C_{w}(x) \varphi(\eta) .
\end{array}
$$

Using the continuity equation we can define stream function $\psi$ such that

$$
u=\frac{\partial \psi}{\partial y}, \quad v=-\frac{\partial \psi}{\partial x}
$$

Integrating (5.5) by using (5.3), we obtain

$$
\psi=K_{1}^{1 / 2}(c+d x)^{(-b+d) / d} F(\eta)
$$

By putting value of $\psi$ from (5.6) into (5.5), then comparing with (5.3) the form of $H(\eta)$ becomes

$$
H(\eta)=K_{1}^{1 / 2}\left[\left(\frac{-b+d}{d}\right) F-b \eta F^{\prime}\right]
$$


Substituting the above new variables in (2.5)-(2.7), we get the following system of ordinary differential equations:

$$
\begin{gathered}
F^{\prime \prime \prime}+K_{1} \frac{(-b+d)}{d} F F^{\prime \prime}-K_{1} \frac{(-2 b+d)}{d} F^{\prime 2}+K_{1} \frac{\mathrm{Gr}}{\mathrm{Re}^{2}} \theta+K_{1} \frac{G c}{\operatorname{Re}^{2}} \varphi=0, \\
\frac{1}{\operatorname{Pr}} \theta^{\prime \prime}+K_{1} \frac{(-b+d)}{d} F \theta^{\prime}-K_{1} \frac{(-4 b+d)}{d} F^{\prime} \theta=0 \\
\frac{1}{\mathrm{Sc}} \varphi^{\prime \prime}+K_{1} \frac{(-b+d)}{d} F \varphi^{\prime}-K_{1} \frac{(-4 b+d)}{d} F^{\prime} \varphi=0
\end{gathered}
$$

and boundary conditions are transformed to

$$
\begin{gathered}
F^{\prime}(0)=1, \quad F(0)=-d^{*} \frac{1}{K_{1}^{1 / 2}((-b+d) / d)}, \quad \theta(0)=1, \\
\varphi(0)=1, \quad F^{\prime}(\infty)=0, \quad \theta(\infty)=0, \quad \varphi(\infty)=0,
\end{gathered}
$$

where Gr $=g \beta T_{0} T_{w}(x) L^{3}(c+d x)^{3} / v^{2}$ is the thermal Grashof number, $\operatorname{Re}=U_{0} u_{w}(x) L(c+$ $d x) / v$ is the Reynold number, and $G c=g \beta T_{0} T_{w}(x) L^{3}(c+d x)^{3} / v^{2}$ is the solutal Grashof number.

In (5.11)

$$
d^{*}=\frac{v_{w}(x)}{U_{0} u_{w}(x)}\left(\frac{U_{0} u_{w}(x) L(c+d x)}{v}\right)^{1 / 2}
$$

By using values of $u_{w}(x)$ and $v_{w}(x)$ in (5.12), we get

$$
d^{*}=\frac{V_{0}}{U_{0}}\left(\frac{U_{0} L}{v}\right)^{1 / 2}
$$

which is a nondimensional constant.

\subsubsection{Example 1}

For $c=0, b=(1-m) / 2, d=1, K_{1}=2 /(m+1)$, and $-4 b+d=n$, the similarity variable and similarity function are

$$
\eta=y\left(\frac{m+1}{2}\right)^{1 / 2}(x)^{(m-1) / 2}, \quad \psi=\left(\frac{2}{m+1}\right)^{1 / 2}(x)^{(m+1) / 2} F(\eta)
$$

Then $u_{w}(x), T_{w}(x)$, and $C_{w}(x)$ take the following form:

$$
u_{w}(x)=x^{m}, \quad T_{w}(x)=x^{n}, \quad C_{w}(x)=x^{n} .
$$


For these variables (5.8)-(5.10) become

$$
\begin{gathered}
F^{\prime \prime \prime}+F F^{\prime \prime}-\frac{2 m}{m+1} F+\frac{2 \lambda}{m+1} \theta+\frac{2 \lambda^{*}}{m+1} \varphi=0, \\
\frac{1}{\operatorname{Pr}} \theta^{\prime \prime}+F \theta^{\prime}-\frac{2 n}{m+1} F^{\prime} \theta=0, \\
\frac{1}{\mathrm{Sc}} \varphi^{\prime \prime}+F \varphi^{\prime}-\frac{2 n}{m+1} F^{\prime} \varphi=0,
\end{gathered}
$$

subject to boundary conditions

$$
\begin{gathered}
F^{\prime}(0)=1, \quad F(0)=-d^{*} \sqrt{\frac{2}{m+1}}, \quad \theta(0)=1, \\
\varphi(0)=1, \quad F^{\prime}(\infty)=0, \quad \theta(\infty)=0, \quad \varphi(\infty)=0,
\end{gathered}
$$

where $\lambda=G r / \operatorname{Re}^{2}$ and $\lambda^{*}=G c / \operatorname{Re}^{2}$. In this case from (5.3) and (5.7) we have

$$
v=-\left(\frac{2}{m+1}\right)^{1 / 2} x^{(m-1) / 2}\left[\left(\frac{m+1}{2}\right) F+\left(\frac{m-1}{2}\right) \eta F^{\prime}\right]
$$

This is all the same as that taken by Ali and Al-Yousef [20] for the problem of mixed convection flow over a stretching surface without considering mass transfer.

\subsubsection{Example 2}

Consider the case for $c=0, b=0, d=1$, and $K_{1}=1$. In this case the form of similarity variable and similarity function is

$$
\eta=y, \quad \psi=x F(\eta)
$$

Therefore, we have

$$
u_{w}(x)=x, \quad T_{w}(x)=x, \quad C_{w}(x)=x,
$$

in consequence of these, (5.8)-(5.10) can be written as

$$
\begin{gathered}
F^{\prime \prime \prime}+F F^{\prime \prime}-F^{\prime 2}+\lambda \theta+\lambda^{*} \varphi=0, \\
\frac{1}{\operatorname{Pr}} \theta^{\prime \prime}+F \theta^{\prime}-F^{\prime} \theta=0, \\
\frac{1}{\mathrm{Sc}} \varphi^{\prime \prime}+F \varphi^{\prime}-F^{\prime} \varphi=0,
\end{gathered}
$$


subject to boundary conditions

$$
\begin{gathered}
F^{\prime}(0)=1, \quad F(0)=F_{w}, \quad \theta(0)=1, \quad \varphi(0)=1, \\
F^{\prime}(\infty)=0, \quad \theta(\infty)=0, \quad \varphi(\infty)=0,
\end{gathered}
$$

where $F_{w}=-d^{*}$. his work.

This problem exactly match to the work done by Yih [21] if we neglect MHD effects in

\subsubsection{Example 3}

In the absence of suction or injection

$$
v_{w}(\tilde{x})=0 \quad \text { at } \tilde{y}=0 .
$$

In such case for $c=0, b=(1-m) / 2, d=1$, and $K_{1}=1$, the similarity variable and similarity function are obtained as

$$
\eta=y(x)^{(m-1) / 2}, \quad \psi=(x)^{(m+1) / 2} F(\eta) .
$$

We then have

$$
u_{w}(x)=x^{m}, \quad T_{w}(x)=x^{2 m-1}, \quad C_{w}(x)=x^{2 m-1},
$$

and (5.8)-(5.10) take the form

$$
\begin{gathered}
F^{\prime \prime \prime}+\left(\frac{m+1}{2}\right) F F^{\prime \prime}-m F^{2}+\lambda \theta+\lambda^{*} \varphi=0, \\
\frac{1}{\operatorname{Pr}} \theta^{\prime \prime}+\left(\frac{m+1}{2}\right) F \theta^{\prime}-(2 m-1) F^{\prime} \theta=0, \\
\frac{1}{\mathrm{Sc}} \varphi^{\prime \prime}+\left(\frac{m+1}{2}\right) F \varphi^{\prime}-(2 m-1) F^{\prime} \varphi=0,
\end{gathered}
$$

together with the boundary conditions

$$
\begin{gathered}
F^{\prime}(0)=1, \quad F(0)=0, \quad \theta(0)=1, \quad \varphi(0)=1, \\
F^{\prime}(\infty)=0, \quad \theta(\infty)=0, \quad \varphi(\infty)=0 .
\end{gathered}
$$

This in fact is the problem considered by Chen [22] neglecting mass transfer. 


\subsubsection{Example 4}

For $b=-2, c=1, d=2$, and $K_{1}=2$. The physical quantities $u_{w}(x), T_{w}(x)$, and $C_{w}(x)$ take the following form

$$
u_{w}(x)=(1+2 x)^{3}, \quad T_{w}(x)=(1+2 x)^{5}, \quad C_{w}(x)=(1+2 x)^{5},
$$

and the similarity variable and functions are

$$
\begin{gathered}
\eta=\frac{y(1+2 x)}{\sqrt{2}}, \quad \psi=\sqrt{2}(1+2 x)^{2} F(\eta), \\
T=T_{w}(x) \theta(\eta), \quad C=C_{w}(x) \varphi(\eta),
\end{gathered}
$$

as a result (5.8)-(5.10) become

$$
\begin{gathered}
F^{\prime \prime \prime}+4 F F^{\prime \prime}-6 F^{\prime 2}+2 \lambda \theta+2 \lambda^{*} \varphi=0, \\
\frac{1}{\operatorname{Pr}} \theta^{\prime \prime}+4 F \theta^{\prime}-10 F^{\prime} \theta=0, \\
\frac{1}{\mathrm{Sc}} \varphi^{\prime \prime}+4 F \varphi^{\prime}-10 F^{\prime} \varphi=0,
\end{gathered}
$$

subject to the boundary conditions

$$
\begin{gathered}
F^{\prime}(0)=1, \quad F(0)=-d^{*}, \quad \theta(0)=1, \\
\varphi(0)=1, \quad F^{\prime}(\infty)=0, \quad \theta(\infty)=0, \quad \varphi(\infty)=0,
\end{gathered}
$$

where $d^{*}$ is the same as given in (5.13).

Such types of stretching velocity, wall temperature, and concentration have not been discussed so far.

\subsection{Case 2: For $d=0$}

In this section, the parameters $b$ and $c$ are taken to be arbitrary and $e=d=\gamma(x)=0$ is considered. This becomes a spiral symmetry where we have considered translation in the variable $x$ and scaling in the variables $y, u, v, T$, and $C$. Setting $b=0$ results in a translational symmetry.

The characteristic equations then are

$$
\frac{d x}{c}=\frac{d y}{b y}=\frac{d u}{-2 b u}=\frac{d v}{-b v}=\frac{d T}{-4 b T}=\frac{d C}{-4 b C} .
$$


The similarity variable is

$$
\eta=\frac{y}{K_{2}^{1 / 2} \exp ((b / c) x)}
$$

and the similarity functions are

$$
\begin{aligned}
& u=\exp \left(-\frac{2 b}{c} x\right) F^{\prime}(\eta), \quad v=\exp \left(-\frac{b}{c} x\right) H(\eta), \\
& T=\exp \left(-\frac{4 b}{c} x\right) \theta(\eta), \quad C=\exp \left(-\frac{4 b}{c} x\right) \varphi(\eta) .
\end{aligned}
$$

From (4.3) and (5.34) we have for $A_{i}=1(i=5,6,7,8)$

$$
\begin{array}{cc}
u=u_{w}(x) F^{\prime}(\eta), & v=-v_{w}(x) H(\eta), \\
T=T_{w}(x) \theta(\eta), & C=C_{w}(x) \varphi(\eta) .
\end{array}
$$

Following the same procedure as done in case 1, we have

$$
\psi=K_{2}^{1 / 2} \exp \left(-\frac{b}{c} x\right) F(\eta)
$$

Note that the variables will be balanced if

$$
H(\eta)=\frac{b}{c} K_{2}^{1 / 2}\left[F+\eta F^{\prime}\right]
$$

In this case, (2.5)-(2.7) will take the following form

$$
\begin{gathered}
F^{\prime \prime \prime}-K_{2} \frac{b}{c} F F^{\prime \prime}+K_{2} \frac{b}{c} F^{2}+K_{2} \frac{\mathrm{Gr}}{\mathrm{Re}^{2}} \theta+K_{2} \frac{G c}{\mathrm{Re}^{2}} \varphi=0, \\
\frac{1}{\operatorname{Pr}} \theta^{\prime \prime}-K_{2} \frac{b}{c} F \theta^{\prime}+4 K_{2} \frac{b}{c} F^{\prime} \theta=0, \\
\frac{1}{\mathrm{Sc}} \varphi^{\prime \prime}-K_{2} \frac{b}{c} F \varphi^{\prime}+4 K_{2} \frac{b}{c} F^{\prime} \varphi=0,
\end{gathered}
$$

subject to the boundary conditions

$$
\begin{gathered}
F^{\prime}(0)=1, \quad F(0)=-d^{*} \frac{1}{K_{2}^{1 / 2}(b / c)}, \quad \theta(0)=1, \quad \varphi(0)=1, \\
F^{\prime}(\infty)=0, \quad \theta(\infty)=0, \quad \varphi(\infty)=0,
\end{gathered}
$$


where the nondimensional physical parameters are

$$
\begin{gathered}
\mathrm{Gr}=\frac{g \beta T_{0} T_{w}(x) L^{3}}{v^{2}}, \quad \operatorname{Re}=\frac{U_{0} u_{w}(x) L}{v}, \\
G c=\frac{g \beta^{*} T_{0} T_{w}(x) L^{3}}{v^{2}}, \\
d^{*}=\frac{v_{w}(x)}{U_{0} u_{w}(x)}\left(\frac{U_{0} u_{w}(x) L}{v}\right)^{1 / 2} .
\end{gathered}
$$

By incorporating (5.34) in (5.41), we get

$$
d^{*}=\frac{V_{0}}{U_{0}}\left(\frac{U_{0} L}{v}\right)^{1 / 2}
$$

Again $d^{*}$ is a nondimensional constant.

\subsubsection{Example 1}

In the case of rigid surface

$$
v_{w}(\tilde{x})=0 \quad \text { at } \tilde{y}=0 .
$$

For $b / c=-1 / 2$ and $K_{2}=2$ the similarity variable and function are

$$
\eta=y\left[\frac{\exp (x)}{2}\right]^{1 / 2}, \quad \psi=[2 \exp (x)]^{1 / 2} F(\eta)
$$

and the surface velocity, temperature, and mass concentration are

$$
u_{w}(x)=\exp (x), \quad T_{w}(x)=\exp (2 x), \quad C_{w}(x)=\exp (2 x) .
$$

With the above functions (5.38) become

$$
\begin{gathered}
F^{\prime \prime \prime}+F F^{\prime \prime}-2 F^{\prime 2}+2 \lambda \theta+2 \lambda^{*} \varphi=0, \\
\frac{1}{\operatorname{Pr}} \theta^{\prime \prime}+F \theta^{\prime}-4 F^{\prime} \theta=0, \\
\frac{1}{\mathrm{Sc}} \varphi^{\prime \prime}+F \varphi^{\prime}-4 F^{\prime} \varphi=0,
\end{gathered}
$$


and boundary conditions are

$$
\begin{gathered}
F^{\prime}(0)=1, \quad F(0)=0, \quad \theta(0)=1, \quad \varphi(0)=1, \\
F^{\prime}(\infty)=0, \quad \theta(\infty)=0, \quad \varphi(\infty)=0,
\end{gathered}
$$

where $\lambda=\mathrm{Gr} / \operatorname{Re}^{2}$ and $\lambda^{*}=G c / \operatorname{Re}^{2}$.

Which is the same as that taken by Partha et al. [23] for mixed convection flow along exponentially stretching surface without considering the mass transfer. In that problem effects of viscous dissipation were examined that are not considered in this paper.

\section{Conclusion}

Mixed convection flow with mass transfer over a stretching surface with suction or injection is studied. Through Lie group analysis symmetries of equations are determined. By applying these similarity variables and functions, original PDEs are converted into ODEs. For specific values of parameters of Lie group transformations, the similarity variables and functions which are available in the literature become special case of our problem. Following the same procedure, now one can easily find the similarity variable and function of his own choice for the particular problem. Specially for stretching problem, use of integral power in boundary conditions is not necessary. Therefore it is a breakthrough in the study of similarity solutions in the sense that only those symmetries that accept restrictions from the boundary conditions are discussed and similarity variables and functions obtained in this way have physical importance. The methodology will help directly to obtain similarity variables and functions of the boundary value problems for any specific boundary value problem at hand.

\section{References}

[1] B. C. Sakiadis, "Boundary-layer behavior on continuous solid surfaces: I. Boundary-layer equationsfor two-dimensional and axisymmetric flow," AIChE Journal, vol. 7, pp. 26-28, 1961.

[2] B. C. Sakiadis, "Boundary-layer behavior on continuous solid surfaces: II. The boundary layer ona continuous flat surface," AIChE Journal, vol. 7, pp. 221-225, 1961.

[3] L. J. Crane, "Flow past a stretching plate," Journal of Applied Mathematics and Physics, vol. 21, pp. 645$647,1970$.

[4] F. K. Tsou, E. M. Sparrow, and R. J. Goldstein, "Flow and heat transfer in the boundary layer on a continuous moving surface," International Journal of Heat and Mass Transfer, vol. 10, no. 2, pp. 219-235, 1967.

[5] V. G. Fox, L. E. Erickson, and L. T. Fan, "Methods for solving the boundary layer equations formoving continuous flat surfaces with suction and injection," AIChE Journal, vol. 14, pp. 726-736, 1969.

[6] L. E. Erickson, L. T. Fan, and V. G. Fox, "Heat and mass transfer on a moving continuous flat plate with suction or injection," Industrial and Engineering Chemistry Fundamentals, vol. 5, no. 1, pp. 19-25, 1966.

[7] P. S. Gupta and A. S. Gupta, "Heat and mass transfer on a stretching sheet with suction or blowing," The Canadian Journal of Chemical Engineering, vol. 55, pp. 744-746, 1977.

[8] V. M. Soundalgekar and T. V. R. Murty, "Heat transfer in MHD flow with pressure gradient, suction and injection," Journal of Engineering Mathematics, vol. 14, no. 2, pp. 155-159, 1980.

[9] L. J. Grubka and K. M. Bobba, "Heat transfer characteristics of a continuous stretching surface with variable temperature," Journal of Heat Transfer, vol. 107, no. 1, pp. 248-250, 1985.

[10] M. E. Ali, "The effect of suction or injection on the Laminar boundary layer development overa stretched surface," ournal of Engineering Sciences King Saud University, vol. 8, pp. 43-58, 1996. 
[11] T. Hayat, M. Mustafa, and I. Pop, "Heat and mass transfer for Soret and Dufour's effect on mixed convection boundary layer flow over a stretching vertical surface in a porous medium filled with a viscoelastic fluid," Communications in Nonlinear Science and Numerical Simulation, vol. 15, no. 5, pp. 1183-1196, 2010.

[12] G. W. Bluman and S. Kumei, Symmetries and Differential Equations, vol. 81 of Applied Mathematical Sciences, Springer, New York, NY, USA, 1989.

[13] N. H. Ibragimov, Ed., CRC Handbook of Lie Group Analysis of Differential Equations, vol. 2, CRC Press, Boca Raton, Fla, USA, 1995.

[14] P. J. Olver, Application of Lie Groups to Differential Equations, Springer, New York, NY, USA, 1989.

[15] L. V. Ovsiannikov, Group Analysis of Differential Equations, Academic Press, New York, NY, USA, 1982.

[16] M. Yürüsoy and M. Pakdemirli, "Exact solutions of boundary layer equations of a special nonNewtonian fluid over a stretching sheet," Mechanics Research Communications, vol. 26, no. 2, pp. 171175, 1999.

[17] M. Yürüsoy, M. Pakdemirli, and Ö. F. Noyan, “Lie group analysis of creeping flow of a second grade fluid," International Journal of Non-Linear Mechanics, vol. 36, no. 6, pp. 955-960, 2001.

[18] S. Sivasankaran, M. Bhuvaneswari, P. Kandaswamy, and E. K. Ramasami, "Lie group analysis of natural convection heat and mass transfer in an inclined surface," Nonlinear Analysis. Modelling and Control, vol. 11, no. 2, pp. 201-212, 2006.

[19] S. Sivasankaran, M. Bhuvaneswari, P. Kandaswamy, and E. K. Ramasami, "Lie group analysis of natural convection heat and mass transfer in an inclined porous surface with heat generation," International Journal Applied Mathematics and Mechanics, vol. 2, pp. 34-40, 2006.

[20] M. Ali and F. Al-Yousef, "Laminar mixed convection boundary layers induced by a linearly stretching permeable surface," International Journal of Heat and Mass Transfer, vol. 45, no. 21, pp. 4241-4250, 2002.

[21] K. A. Yih, "Free convection effect on MHD coupled heat and mass transfer of a moving permeable vertical surface," International Communications in Heat and Mass Transfer, vol. 26, no. 1, pp. 95-104, 1999.

[22] C.-H. Chen, "Laminar mixed convection adjacent to vertical, continuously stretching sheets," Heat and Mass Transfer, vol. 33, no. 5-6, pp. 471-476, 1998.

[23] M. K. Partha, P. V. S. N. Murthy, and G. P. Rajasekhar, "Effect of viscous dissipation on the mixed convection heat transfer from an exponentially stretching surface," Heat and Mass Transfer/Waerme, vol. 41, no. 4, pp. 360-366, 2005. 


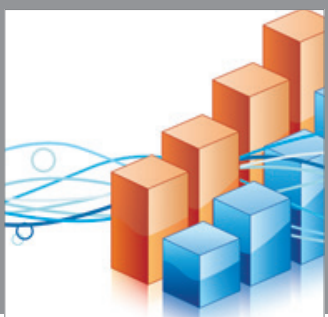

Advances in

Operations Research

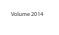

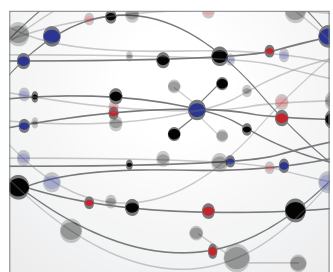

\section{The Scientific} World Journal
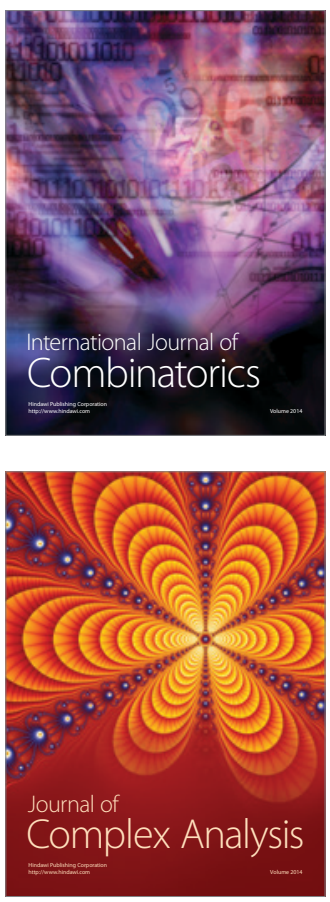

International Journal of

Mathematics and

Mathematical

Sciences
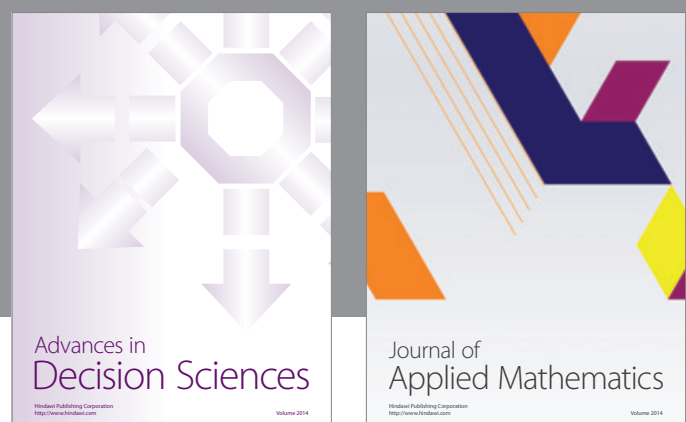

Journal of

Applied Mathematics
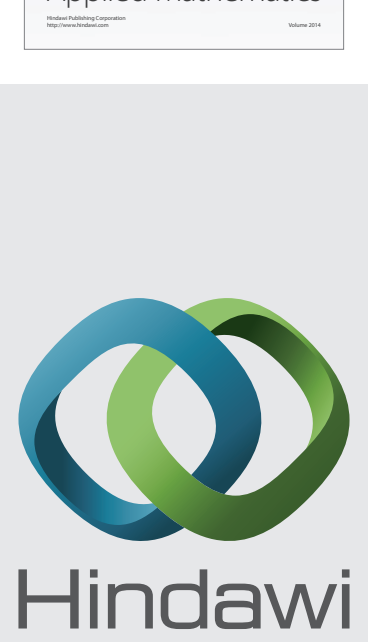

Submit your manuscripts at http://www.hindawi.com
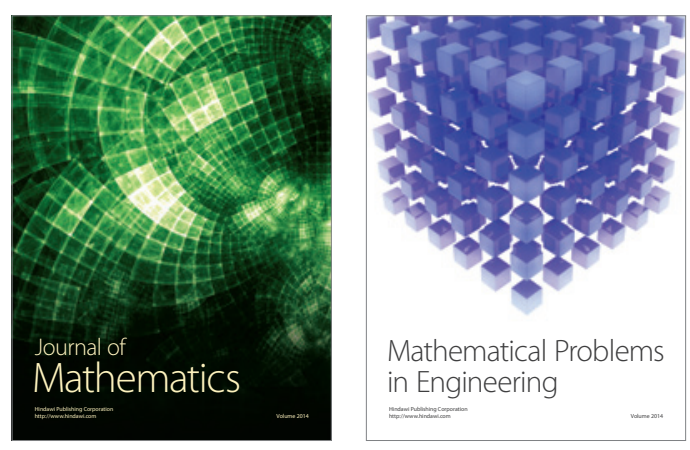

Mathematical Problems in Engineering
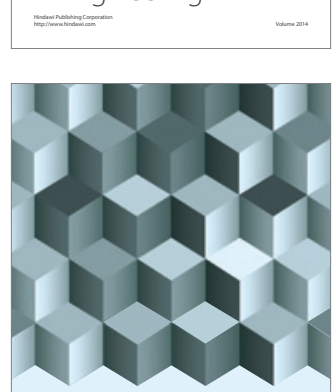

Journal of

Function Spaces
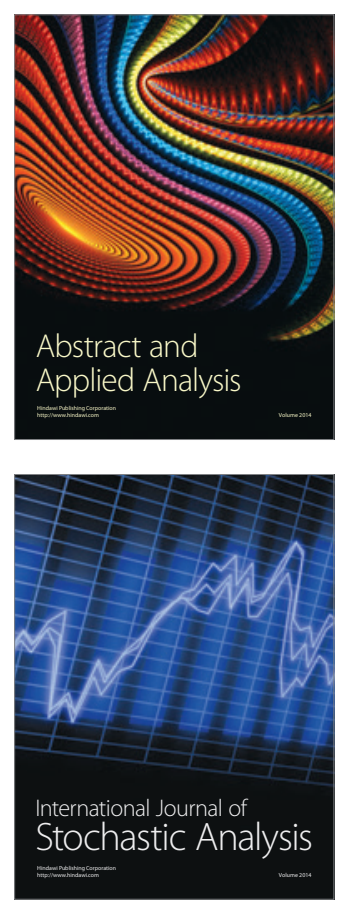

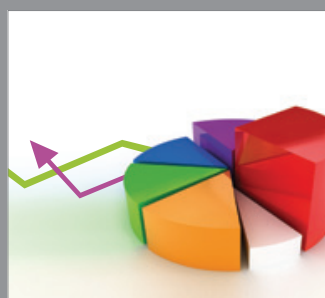

ournal of

Probability and Statistics

Promensencen
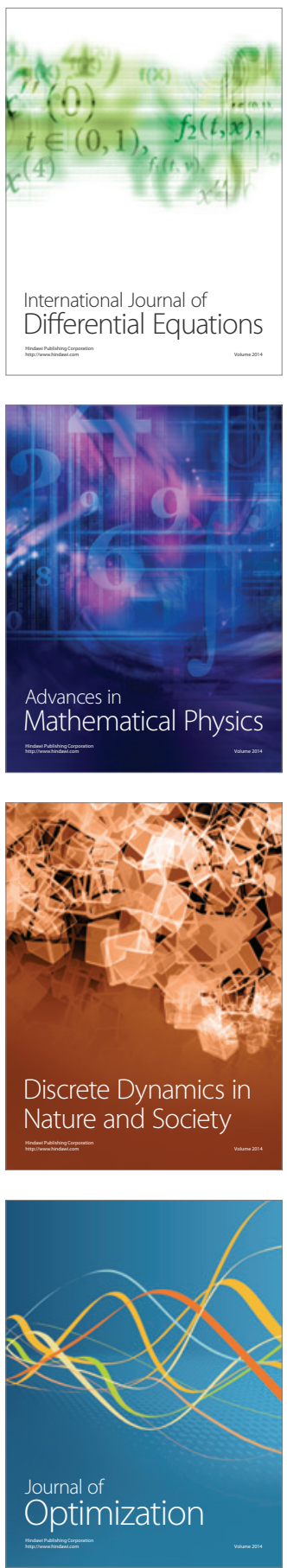